\title{
Caracterización de funciones frontales y ejecutivas en pacientes heroinómanos en tratamiento con metadona
}

\author{
Characterization of frontal and executive functions \\ in heroin patients in methadone treatment
}

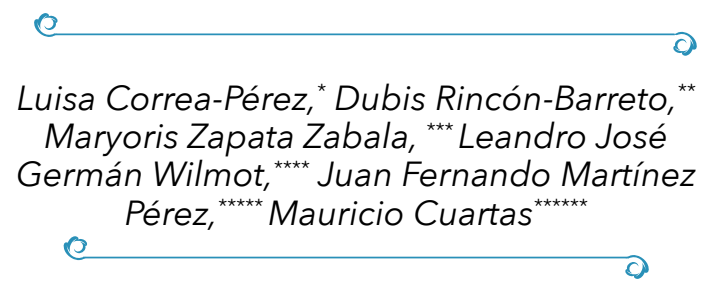

Recibido 31. 01. 2020 • Arbitrado 15. 02. $2020 \bullet$

Aprobado 25.02. 2020

* Facultad de Psicología y Ciencias Sociales, Universidad Católica Luis Amigó. Medellín, Colombia. https:/ / orcid.org/0000-0001-5053-691X. luisa.correape@amigo.edu.co

* Facultad de Psicologia y Ciencias Sociales, Universidad Católica Luis Amigó. Medellín, Colombia. https:// orcid.org/0000-0002-8322-889X. dubis.rinconba@amigo.edu.co

*** Grupo de psicología y Neurociencias, Facultad de Psicología, Universidad de San Buenaventura. Medellín, Colombia. https:/ / orcid.org/0000-00015734-0928, maryoris.zapata@usbmed. edu.co

**** Coordinación de la Maestría de Salud Pública; Dirección Departamento de Acreditación, Universidad Central del Este Republica Dominicana, https:// orcid.org/0000-001-70903043.1german@uce.edu.do

***** Graduate School of Education. Ana G. Mendez University. Puerto Rico. https:// orcid.org/0000-0001-5190-0963. martinezj11@suagm.edu

****** Estudios del Comportamiento, Departamento de Psicología, Escuela de Humanidades, Universidad EAFIT, Medellín, Colombia, http://orcid.org/0000-0001-9007-713X, jmcuartasa@ eafit.edu.co

\section{Resumen}

Con el propósito de determinar las características de las funciones frontales y ejecutivas en pacientes heroinómanos, hombres (n: 45) en tratamiento con metadona de la E.S.E Hospital Carisma en Antioquia, se diseña un estudio observacional descriptivo, y se aplican el Test Breve de Inteligencia de Kaufman y la Batería Neuropsicológica de las Funciones Ejecutivas y Lóbulos Frontales (BANFE). Los resultados permitieron establecer que el funcionamiento ejecutivo en estos pacientes presenta en la mayoría $(n=66,6 \%)$ una alteración severa en tareas de autocontrol, toma de decisiones y regulación emocional, asimismo, se evidencia alteración en las áreas dorsolateral, orbitofrontal y frontomedial.

Palabras clave: heroína, metadona, funciones frontales y ejecutivas. 


\section{Abstract}

In order to determine the characteristics of frontal and executive functions in male heroin users (n: 45) treated with methadone at the State Health-care Institution (ESE, Empresa Social del Estado, for its abbreviation in Spanish) Carisma Hospital in Antioquia, a descriptive observational study is carried out using both the Kaufman Brief Intelligence Test, and the Batería Neuropsicológica de Funciones Ejecutivas y Lóbulos Frontales (BANFE) test. The results showed that executive functioning in most of these patients presents a severe alteration $(n=66.6 \%)$ concerning self-control tasks, decision making, and emotional regulation. In addition, some alterations in the dorsolateral, orbitofrontal and frontomedial areas are observed.

Keywords: heroin, methadone, executive functions.

\section{Introducción}

Las drogas se pueden clasificar según diferentes criterios, uno de ellos es por el efecto que generan en sistema nervioso. Desde este criterio se encuentran las drogas estimulantes, alucinógenas, mixtas o depresoras. Para que estas generen adicción el sujeto debe atravesar una escala de consumo que va desde el uso, abuso, dependencia hasta la adicción. Según el informe mundial de la salud, publicado en el año 2014, el consumo de drogas problemático, afecta entre 16 y los 39 millones de personas, por consumos habituales y trastornos de consumo o dependencia (Ministerio de Justicia y del Derecho, 2014).

Según Ministerio de Justicia y del Derecho (2015) se estima que Medellín - Colombia tiene la mayor tasa de consumo con el 8.2\%, seguido por Quindío y Risaralda, con prevalencias superiores al 6\%; Antioquia y Meta que superan el $5 \%$. Además, de acuerdo a las tasas de consumo en el último año, se estiman las siguientes cifras globales de consumidores en cada grupo de edad: 159 mil adolescentes de 12 a 17 años, 347 mil jóvenes de 18 a 24 años y 216 mil en el grupo 25 a 34 años. Al analizar la tendencia por edad, es claro que ésta incrementa en forma continua, y se relaciona con conductas de riesgo. Aproximadamente en la mitad de los accidentes mortales el conductor o el peatón están bajo el efecto de una intoxicación. Además, el 10\% de los sujetos con dependencia a consumo se suicida, debido a que el estado del ánimo, con frecuencia, puede ser inducido por el uso de sustancias (Colombia, Ministerio de la Protección Social, 2010).

Por su parte, la heroína es una sustancia ilegal altamente adictiva dada la existencia de receptores cerebrales opioides, cuyo mecanismo de acción favo- 
rece la respuesta de placer de forma casi inmediata posterior a su consumo (Oficina de las Naciones Unidas contra la Droga y el Delito, 2015). A nivel neuropsicológico, Wang et al. (2015) afirman que las personas adictas a la heroína presentan enlentecimiento en la velocidad del procesamiento y un déficit en la evaluación e inhibición de conductas inapropiadas, además de un alto riesgo de una sobredosis o de morir, de contraer VIH y otras enfermedades, lo cual puede ocurrir por el intercambio de agujas u otros equipos de inyección.

De otro lado, la metadona es un agente sintético que se encarga de ubicarse en dichos receptores cerebrales afectados por la heroína; una de sus principales funciones es aliviar la ansiedad de consumo y los síntomas de abstinencia físicos y orgánicos que produce la sustancia (Darke, McDonald, Kaye \& Torok, 2012; Liao, et al, 2014). Con este objetivo y, además, pensando en la disminución de riegos, se creó el programa de tratamiento de mantenimiento con metadona (Bobes y Bobes, 2012), también conocido como terapia de sustitución. La terapia de mantenimiento con metadona (TMM), se constituye en un elemento importante dentro del tratamiento de la dependencia de opioides. En esta terapia se utiliza metadona durante un periodo de tiempo variable, según la duración del tratamiento, con tres fines clínicos: suprimir los síntomas de abstinencia, disminuir la ansiedad de consumo y bloquear los efectos de opioides, si se llegasen a consumir. Igualmente, se persigue mejorar la adherencia con el tratamiento, mejorar la calidad de vida, disminuir el riesgo de infección (VIH, hepatitis B y C) y de sobredosis, entre otros (Colon \& Duro, 2009, citados por Generalitat de Catalunya, Departamento de Salud, 2009).

En la guía de tratamiento de Opiodes (E.S.E. Hospital Carisma, 2013), definen la Metadona como un agonista sintético de la heroína, que cuenta con buena biodisponibilidad y se suministra por vía oral. Este es el agente más utilizado y con mayor evidencia y eficacia en los programas de abstinencia a opiáceos, debe ser utilizado bajo supervisión médica. El tratamiento con metadona está estrictamente regulado debido a que induce la dependencia a opiáceos. Según la Asociación Nacional de Medicamentos, Alimentos y Tecnología Médica ANMAT (2014), las dosis óptimas de metadona son entre 60$100 \mathrm{mg}$, establecen las dosis de la siguiente manera: dosis bajas (10-30 mg), dosis moderadas (40-70 mg), dosis altas (80-100 mg).

Según la OMS (2009), la terapia de mantenimiento con agonistas opioides fue encontrada como la más efectiva, reportando beneficios como: supresión de los síntomas de abstinencia, reducción de la ansiedad de consumo (Cravings) y disminución de los efectos euforizantes de los opioides de corta acción.

Respecto al funcionamiento neuropsicológico en pacientes en mantenimiento con metadona frente heroinómanos abstinentes, Verdejo et al. (2005) 
señalan que los pacientes pertenecientes al MMP mostraron un rendimiento significativamente más lento en la velocidad de procesamiento, atención visuoespacial y las pruebas de flexibilidad cognitiva, menos precisión en la memoria de trabajo y pruebas de razonamiento analógico extraído de la Escala Wechsler de Inteligencia para Adultos. Según Prosser et al. (2006), tienen un pobre desempeño en las tareas de función verbal, visual-análisis espacial, la memoria, y la resistencia a la distracción, sugiriendo deterioro neuropsicológico en los receptores de mantenimiento con metadona (MMT) a largo plazo. Los pacientes en terapia de mantenimiento, de acuerdo con Darke et al. (2012) demuestran déficit en el rendimiento cognitivo, independientemente del tipo de medicamento.

En forma complementaria a los resultados de las anteriores investigaciones, Brand, Roth-Bauer, Driessen \& Markowitsch (2008) y Pau, Lee \& Chan, (2002) plantean que este deterioro en las funciones ejecutivas afecta de manera directa el funcionamiento emocional y la toma de decisiones. En el caso de la terapia desarrollada en E.S.E Hospital Carisma, se trata de un programa completo que incluye una intervención interdisciplinaria desde la visión médica, la psicoterapia, la intervención familiar y la terapia ocupacional, el cual ha demostrado reducir la morbilidad y mortalidad asociada a la adicción a heroína. No obstante, existen vacíos en la investigación relacionados con el rendimiento cognitivo de los pacientes bajo tratamiento con metadona, por lo que esta investigación tuvo como objetivo caracterizar las funciones frontales y ejecutivas de los pacientes heroinómanos que se encuentran adscritos al programa de mantenimiento con metadona, de la Empresa Social del Estado - E.S.E Hospital Carisma en la ciudad de Medellín - Colombia.

\section{Método}

\section{Participantes}

La investigación se realizó con hombres mayores de 18 años, pertenecientes al Programa de mantenimiento con metadona (P.M.M). La muestra quedó compuesta por 45 pacientes, con diagnóstico de adicción a la heroína. Los participantes han recibido tratamiento intrahospitalario de rehabilitación y asisten al P.M.M, algunos de ellos tenían consumos activos de otras sustancias psicoactivas.

Como criterios de inclusión se consideraron: que fueran hombres adscritos al Programa de mantenimiento con metadona con seguimiento periódico por toxicología y/o psiquiatría, pacientes con CI promedio (70), con habilidades 
de lecto-escritura. Se excluyeron todos aquellos pacientes con diagnóstico dual o alteraciones neurológicas, preexistencia de diagnóstico de enfermedad inmunológica como VIH y consumo de opiáceos diferentes a la heroína.

\section{Instrumentos}

International Neuropsychiatric Interview - MINI: es una entrevista diagnóstica estructurada, de breve duración, que explora los principales trastornos psiquiátricos del Eje I del DSM-IV y la CIE-10.

Batería Neuropsicológica de Funciones Ejecutivas y Lóbulos Frontales (BANFE): desarrollada por Flores, Ostrosky-Solís \& Lozano (2014). Este instrumento evalúa con 15 subpruebas las funciones ejecutivas vinculadas al funcionamiento de los lóbulos frontales. Las subpruebas de esta batería valoran cada una de las siguientes áreas cerebrales: corteza prefrontal dorsolateral, corteza prefrontal anterior, corteza orbitofrontal, corteza frontomedial.

Test Breve de Inteligencia de Kaufman (K-BIT): está diseñado para la medida de la inteligencia general de individuos, tiene un amplio rango de edad (desde los 4 a los 90 años). Suministra un C.I. verbal, un C.I. no verbal y un C.I. compuesto que resume el rendimiento total en el test. Esta prueba está constituida por dos subtest: vocabulario y matrices.

\section{Procedimiento}

Una vez que se obtuvo el consentimiento informado por escrito, se administró la entrevista MINI y se valoró el coeficiente intelectual con el K-BIT excluyendo a aquellos participantes con un $\mathrm{CI}$ inferior a 70. Posteriormente, se realizó la aplicación de la BANFE, para evaluar el funcionamiento ejecutivo en los pacientes participantes. Para el análisis de los datos se emplearon medidas de tendencia central, como la Media (M) y Desviación Estándar (DE)

\section{Resultados}

Se halló que los pacientes evaluados, todos de sexo masculino, solteros en mayor porcentaje $(66,6 \%)$, presentan características sociales similares. De acuerdo con las variables sociodemográficas, sus edades están comprendidas entre los 22 y 43 años, con una $M(29,8)$ y $D E(4,8)$; sus estudios son de una totalidad de once años, grado de escolaridad bachillerato. La mayoría de ellos tienen trabajos informales (34,7\%), seguidos de aquellos que no tienen ninguna ocupación (29\%), solo el $13 \%$ de los pacientes evaluados se encuentra 
estudiando y el 23, 9\% tiene un trabajo de carácter formal, mayoritariamente de un estrato bajo, con edades tempranas de inicio entre los 13 y 15 años, con una $M(15,3)$ y $D E(2,6)$ (Ver tabla 1$)$.

Tabla 1. Descripción de las variables sociodemográficas

\begin{tabular}{lcc}
\hline \multicolumn{1}{c}{ Variables } & n & \% \\
\hline Ocupación & & \\
\hline Formal & 11 & 23,9 \\
Informal & 16 & 34,7 \\
Sin ocupación & 13 & 29,5 \\
Estudiante & 6 & 13 \\
Estado civil & & \\
Soltero & 41 & 89,1 \\
Separado & 1 & 2,1 \\
Unión libre & 4 & 8,6 \\
\hline Estrato socioeconómico & & \\
\hline Bajo & 30 & 66,6 \\
Medio & 13 & 28,8 \\
Alto & 2 & 4,4 \\
\hline \multicolumn{2}{c}{ M } & DE \\
\hline Edad & 29,8 & 4,8 \\
Años de escolaridad & 11,1 & 2,0 \\
\hline
\end{tabular}

Fuente: Elaboración propia.

En cuanto a las características de las variables clínicas se resalta el policonsumo en la mayoría de ellos, prevalece la dependencia y consumo activo de la cocaína $(30,4 \%)$, seguido del consumo de THC (26\%) y el consumo de ambas sustancias (cocaína y THC) en el mismo porcentaje anterior (26\%). Los resultados de la entrevista psiquiátrica dan cuenta de que el 47,8\% de los pacientes que pertenecen al P.M.M., presenta un riesgo suicida leve, y solo el $8,6 \%$ cumplen con los criterios de trastornos psiquiátricos, especialmente el de angustia y personalidad antisocial.

Todos los participantes han tenido preinscritos tratamientos farmacológicos; la metadona es el medicamento común entre ellos, con dosis variantes entre bajas - moderadas $(45,6 \%)$ y altas $(54,3 \%)$, según lo indicado por el médico tratante, el $26,6 \%$ tiene dentro de su tratamiento médico el uso de antipsicóticos, el 66,6\% utiliza antidepresivos, el 22,2\% estabilizadores del ánimo, el 20\% agonista de la nicotina y el 17\% usa antiepilépticos (Ver Tabla 2). 
Tabla 2. Características clínicas

\begin{tabular}{|c|c|c|}
\hline Variables & $\mathbf{M}$ & DE \\
\hline Edad de inicio de consumo & 15,3 & 2,6 \\
\hline Años de consumo & 14,4 & 5,7 \\
\hline C.I & 87,1 & 8,6 \\
\hline MINI & n & $\%$ \\
\hline \multicolumn{3}{|l|}{ Riesgo suicida } \\
\hline Leve & 22 & 47,8 \\
\hline Alto & 2 & 4,34 \\
\hline \multicolumn{3}{|l|}{ Dependencia } \\
\hline THC & 12 & 26 \\
\hline Cocaína & 14 & 30,4 \\
\hline Coc у THC & 12 & 26 \\
\hline Inhalantes & 1 & 2,1 \\
\hline Inhalantes y Coc & 2 & 4,3 \\
\hline \multicolumn{3}{|l|}{ Trastornos } \\
\hline Angustia & 1 & 2,1 \\
\hline Personalidad & 3 & 6,5 \\
\hline Consumo activo & 15 & 32,6 \\
\hline \multicolumn{3}{|l|}{ Medicamentos } \\
\hline Antipsicótico & 12 & 26,6 \\
\hline Antidepresivo & 30 & 66,6 \\
\hline Estabilizador del animo & 10 & 22,2 \\
\hline Agonista de la nicotina & 9 & 20 \\
\hline Antiepiléptico & 8 & 17,7 \\
\hline \multicolumn{3}{|l|}{ Metadona } \\
\hline Dosis baja-moderada & 21 & 45,6 \\
\hline Dosis alta & 25 & 54,3 \\
\hline
\end{tabular}

Fuente: Elaboración propia.

En cuanto al desempeño ejecutivo y las características de las funciones ejecutivas, los pacientes presentan alteraciones entre leves - moderadas $(13,6 \%)$ y severas $(66,6 \%)$, pocos presentan normalidad (20\%) en el funcionamiento de las funciones ejecutivas, lo cual puede traducirse en dificultades en el autocontrol, la toma de decisiones y la regulación emocional. Asimismo, los resultados en las subpruebas del área orbitomedial dan cuenta de que los pacientes poseen alteración severa en un 44,4\% (n: 20); el 40\% (n: 18) un desempeño normal y el 15,5 (n: 7) alteración leve-moderada, lo cual puede 
representar dificultades en la regulación emocional, en los estados afectivos, en la detección de riesgos y beneficios al momento de tomar decisiones.

Con relación al área dorsolateral y de acuerdo con los resultados, un 40\% de los pacientes evaluados tiene un desempeño normal en las funciones de planeación, memoria de trabajo, solución de problemas, flexibilidad mental, estrategias de trabajo y secuenciación, sin embargo, es necesario resaltar que se presentaron alteración severa $(35,5 \%)$ y alteración leve-moderada $(22,2 \%)$. Hay que anotar que el 60\% de pacientes evaluados en esta investigación (n: 27), presentan alteración severa en estas funciones, el 26,6\% desempeño normal (n: 12), el 8,8\% (n: 4) alteración leve-moderada y solo el 4,3\% un rendimiento normal alto (n: 2) (Ver tabla 3).

Tabla 3. Desempeño BANFE por áreas

\begin{tabular}{lcc}
\hline \multicolumn{1}{c}{ Área } & M & DE \\
\hline Orbitomedial & 86,3 & 15,2 \\
Normal & $\boldsymbol{n}$ & $\boldsymbol{\%}$ \\
Alteración leve-moderada & 7 & 40 \\
Alteración severa & 20 & 15,5 \\
\hline Dorsolateral & 80,4 & 16,4 \\
& $\boldsymbol{n}$ & $\mathbf{\%}$ \\
Normal & 18 & 40 \\
Normal alto & 1 & 2,2 \\
Alteración leve-moderada & 10 & 22,2 \\
Alteración severa & 16 & 35,5 \\
\hline Frontal & 93,0 & 15,2 \\
& $\boldsymbol{n}$ & \% \\
Normal & 12 & 26,6 \\
Normal alta & 2 & 4,3 \\
Alteración leve-moderada & 4 & 8,8 \\
Alteración severa & 27 & 60 \\
\hline
\end{tabular}

Fuente: Elaboración propia.

En general, el desempeño de las funciones ejecutivas, detallado en la Tabla 4, expresa que el 66,6\% de los sujetos evaluados (n: 30) presenta alteración severa en las funciones de fluidez verbal, productividad, flexibilidad mental, planeación visoespacial y secuencial, secuenciación inversa y control de codificación. El 20\% presentó una ejecución normal y el 13,3 \% alteración levemoderada. 
Tabla 4. Desempeño BANFE (Funciones ejecutivas)

\begin{tabular}{lcc}
\hline & $\boldsymbol{M}$ & $\boldsymbol{D E}$ \\
\hline Funciones Ejecutivas & 78,9 & 14,7 \\
& $\boldsymbol{n}$ & $\boldsymbol{\%}$ \\
\cline { 2 - 3 } Normal & 9 & 20 \\
Alteración leve-moderada & 6 & 13,3 \\
Alteración severa & 30 & 66,6 \\
\hline
\end{tabular}

Fuente: Elaboración propia.

\section{Discusión y conclusiones}

A partir de los resultados de las características clínicas y en coherencia con lo estudiado por Isaza, Suárez, Henao y González (2010), se evidencia una disminución en la edad de inicio de consumo de sustancias psicoactivas, que empieza en la adolescencia, ligado a las conductas de riesgo que las personas asumen en esa etapa del desarrollo.

En la evaluación realizada a los 45 pacientes participantes, se halló que las áreas evaluadas mediante la batería seleccionada (BANFE) presentan alteraciones que oscilan entre leve-moderada y severa, además de un coeficiente intelectual limítrofe, que genera la hipótesis de un deterioro cognitivo articulado al consumo de sustancias psicoactivas. Los participantes en su mayoría presentan dificultades en la capacidad para inhibir respuestas erradas y evitar el uso de estas de manera repetitiva, aunado a la escasa capacidad para mantener una conducta por medio de reforzamientos positivos (Martín-Contero, Secades-Villa y Tirapu-Ustárroz, 2012; Tirapu, García-Molina, Ríos-Lago \& Ardila, 2012).

Algunos estudios con relación al consumo de heroína y metadona, han abordado cambios en las funciones ejecutivas (Gupta et al., 2014; Liao et al., 2014; Sittambalam, Vij, \& Ferguson, 2014; Wang et al., 2015), señalan que el consumo de metadona se asocia con un peor rendimiento en las medidas de procesamiento sensorial, velocidad psicomotora, atención dividida y la memoria de trabajo. Los efectos de la dosis más alta de Metadona, se asocian con un peor rendimiento en los aspectos de la atención y la memoria de trabajo.

Por su parte, Ardila y Ostrosky-Solís (2008), definen la función ejecutiva como la encargada de la metacognicion, la solución de problemas, planeación, formación de conceptos, desarrollo e implementación de estrategias, así como de la memoria de trabajo; funciones especialmente afectadas por el consumo de heroína. 
Considerando lo anterior, los pacientes consumidores que participaron en este estudio, mostraron características similares a los sujetos con lesiones en la corteza orbitofrontal, quienes presentan la tendencia a seleccionar la recompensa inmediata a pesar de las consecuencias negativas, afectando considerablemente la toma de decisiones en el diario vivir de estos sujetos (Cummings, 1993; Verdejo-García, Pérez-García \& Bechara, 2006).

De igual manera, estos pacientes presentaron dificultad en la regulación de emociones, especialmente en situaciones de estrés y ansiedad, déficit en el control de impulsos, escasa habilidad para mantener una actitud optimista que le permita interactuar con el ambiente para generar emociones positivas y pensamientos automotivadores (Pineda, 2000; Ardila, 2013).

También, los hallazgos de esta investigación son coincidentes con lo descrito por Yan et al. (2014), quienes realizaron un estudio comparativo entre adictos a la heroína y controles sanos y en el cual encontraron que los sujetos consumidores presentaron un peor desempeño en tareas que implicaban memoria de trabajo y rendimiento en toma de decisiones. Se obtiene concordancia con la evaluación sesgo atencional en pacientes consumidores de heroína, realizada por Constantinou et al. (2010), quienes encontraron lentitud en las respuestas de las pruebas y dificultades para dirigir la atención relacionada con estados emocionales y situaciones de estrés. Adicional a las dificultades atencionales, el estudio refiere dificultades en la evaluación del rendimiento atencional, la atención selectiva y el control ejecutivo de la acción con la prueba de Go/no Go. Los investigadores reportaron que los pacientes con metadona tuvieron un desempeño inferior en las pruebas atencionales (Rapeli, Fabritius, Kalska \& Alho, 2011).

En síntesis, los resultados de esta investigación permiten establecer una caracterización del funcionamiento ejecutivo, a partir del levantamiento del perfil neuropsicológico de los consumidores de opiáceos, en el que se destaca las dificultades en la memoria a corto plazo, en memoria verbal y visual demorada, atención y concentración, habilidades motoras finas, habilidades visoespaciales y visomotoras y fluencia verbal, y a largo plazo, deterioro en funciones ejecutivas y razonamiento abstracto (Fernández-Duque, Baird, \& Posner, 2000; Verdejo-García, López-Torrecillas, Orozco, \& Pérez, 2002). En la descripción de las variables apreciadas en este estudio, debe advertirse que el grupo de pacientes adictos participantes representa un porcentaje de los inscritos al P.M.M, por ende, los hallazgos proporcionan un panorama de estas características, además de la necesidad de generar nuevas estrategias de intervención. 


\section{Referencias}

Ardila, A. (2013). Función Ejecutiva [fundamentos y evaluación]. Miami: Florida International University. Recuperado de https://goo.gl/YEzXoD

Ardila, A., Ostrosky-Solís, F. (2008). Desarrollo Histórico de las Funciones Ejecutivas. Revista Neuropsicología, Neuropsiquiatría y Neurociencias, 8(1), 1-21. Recuperado de https://dialnet.unirioja.es/servlet/articulo? codigo $=3987433$

Asociación Nacional de Medicamentos, Alimentos y Tecnología Médica (ANMAT). (2014). Disposición $n^{\circ}$ 4790. Recuperado de http://www.anmat.gov.ar/boletin_ anmat/Julio_2014/Dispo_4790-14.pdf

Bobes, J., Bobes, M. T. (2012). Efectividad a largo plazo de los tratamientos de mantenimiento con metadona en personas con adicción a derivados opiáceos. Adicciones, 24(3), 179-184. Recuperado de http://www.adicciones.es/index. $\mathrm{php} /$ adicciones/article/view/87/86

Brand, M., Roth-Bauer, M., Driessen, M., Markowitsch, H. J. (2008). Executive functions and risky decision-making in patients with opiate dependence. Drug and Alcohol Dependence, 97(1-2), 64-72. DOI: http://doi.org/10.1016/j. drugalcdep.2008.03.017

Constantinou, N., Morgan, C., Battistella, S., O'Ryan, D., Davis, P., y Curran, H. V. (2010). Attentional bias, inhibitory control and acute stress in current and former opiate addicts. Drug and Alcohol Dependence, 109(1), 220-225. DOI: http://doi. org/10.1016/j.drugalcdep.2010.01.012

Cummings, J. L. (1993). Frontal-subcortical circuits and human behavior. Archives of Neurology, 50(8), 873-880. Recuperado de https://www.ncbi.nlm.nih.gov/ pubmed/8352676

Darke, S., McDonald, S., Kaye, S., Torok, M. (2012). Comparative patterns of cognitive performance amongst opioid maintenance patients, abstinent opioid users and non-opioid users. Drug and Alcohol Dependence, 126(3), 309-315. DOI: http:// doi.org/10.1016/j.drugalcdep.2012.05.032

E.S.E. Hospital Carisma. (2013). Guía de atención integral para los problemas relacionados con heroína y otros opioides [documento inédito]. Medellín, Colombia: E.S.E. Hospital Carisma.

Fernández-Duque, D., Baird, J. A., Posner, M. I. (2000). Executive Attention and metacognitive regulation. Consciousness and Cognition, 9(2), 288-307. DOI: http://doi.org/10.1006/ccog.2000.0447 
Flores, J. C., Ostrosky-Solís, F., Lozano, A. (2014). Batería neuropsicológica de Funciones Ejecutivas y Lóbulos Frontales. México: Manual Moderno.

Generalitat de Catalunya. Departamento de Salud. (2009). Tratamiento de mantenimiento con metadona: manual de práctica clínica. Barcelona: Generalitat de Catalunya. Departamento de Salud. Recuperado de http://drogues.gencat. cat/es/detalls/Article/Manual-de-practica-clinica-sobre-els-tractaments-demanteniment-amb-metadona

Gupta, S., Iudicello, J. E., Shi, C., Letendre, S., Knight, A., Li, J., ... Heaton, R. K. (2014). Absence of neurocognitive impairment in a large Chinese sample of HCVinfected injection drug users receiving methadone treatment. Drug and Alcohol Dependence, 137, 29-35. DOI: http://doi.org/10.1016/j.drugalcdep.2013.12.021

Isaza, C., Suárez, P., Henao, J., González, M. (2010). Características demográficas y de consumo en pacientes adictos a heroína o derivados de la coca. Investigaciones Andina, 12(21), 24-34. Recuperado de http://www.scielo.org.co/scielo. php?script=sci_arttext\&pid=S0124-81462010000200003

Liao, D-L., Huang, C-Y., Hu, S., Fang, S-C., Wu, C-S., Chen, W-T., ... Li, C. S. (2014). Cognitive Control in Opioid Dependence and Methadone Maintenance Treatment. PLoS One, 9(4), DOI: http://doi.org/10.1371/journal.pone.0094589

Martín-Contero, M. C., Secades-Villa, R., Tirapu-Ustárroz, J. (2012). Cognición social en adictos a opiáceos. Revista de Neurología, 55(12), 705-712. Recuperado de https://www.neurologia.com/articulo/2012553

Ministerio de Justicia y del Derecho. (2015). La heroína en Colombia: producción, uso e impacto en la salud pública: análisis de la evidencia y recomendaciones de política. Bogotä: Observatorio de Drogas de Colombia. Recuperado de http:// www.odc.gov.co/Portals/1/publicaciones/pdf/consumo/estudios/nacionales/ C003132015-la_heroina_en_colombia_produccion_impacto_salud.pdf

Ministerio de Justicia y del Derecho, Observatorio de Drogas de Colombia y Ministerio de Salud y Protección Social. (2014). Estudio nacional de consumo de sustancias psicoactivas en Colombia 2013: informe final. Bogotá: Ministerio de Justicia y del Derecho. Recuperado de https://www.unodc.org/documents/colombia/2014/ Julio/Estudio_de_Consumo_UNODC.pdf

Ministerio de la Protección Social. (2010). Situación de salud mental del adolescente: Estudio Nacional de Salud Mental Colombia. Bogotá: Ministerio de la Protección Social. Recuperado de https://revistas.javeriana.edu.co/index.php/ imagenydesarrollo/article/view/1635/1056

Oficina de las Naciones Unidas contra la Droga y el Delito. (2015). Informe mundial sobre las drogas 2015: resumen ejecutivo. Vienna: United Nations Office On Drugs 
and Crime (UNODC). Recuperado de https://www.unodc.org/documents/ wdr2015/World_Drug_Report_2015_Spanish_pdf

Organización Mundial de la Salud (2009). Guidelines for the psychosocially assisted pharmacological treatment of opioid dependence. Geneva, Switzerland: World Health Organization.

Pau, C. W., Lee, T. M., Chan, S. F. (2002). The impact of heroin on frontal executive functions. Archives of Clinical Neuropsychology, 17(7), 663-670. DOI: http://doi. org/10.1016/S0887-6177(01)00169-X

Pineda, D. A. (2000). La Función Ejecutiva y sus trastornos. Revista de Neurología, 30(8), 764-768. DOI: https://doi.org/10.33588/rn.3008.99646

Prosser, J., Cohen, L., Steinfeld, M., Eisenberg, D., London, E., Galynker, I. (2006). Neuropsychological functioning in opiate-dependent subjects receiving and following methadone maintenance treatment. Drug and Alcohol Dependence, 84(3), 240-247. DOI: http://doi.org/10.1016/j.drugalcdep.2006.02.006

Rapeli, P., Fabritius, C., Kalska, H., Alho, H. (2011). Cognitive functioning in opioid-dependent patients treated with buprenorphine, methadone, and other psychoactive medications: stability and correlates. BMC Clinical Pharmacology, 11(13), 1-15. DOI: http://doi.org/10.1186/1472-6904-11-13

Sittambalam, C. D., Vij, R., Ferguson, R. (2014). Buprenorphine Outpatient Outcomes Project: can Suboxone be a viable outpatient option for heroin addiction? Journal of Community Hospital Internal Medicine Perspectives, 4(2), 1-6. DOI: http://doi. org/10.3402/jchimp.v4.22902

Tirapu, J., García-Molina, A., Ríos-Lago, M., Ardila, A. (2012). Neuropsicología de la corteza prefrontal y funciones ejecutivas. Barcelona: Viguera.

Verdejo-García, A., López-Torrecillas, F., Orozco, C., Pérez, M. (2002). Impacto de los deterioros neuropsicológicos asociados al consumo de sustancias sobre la práctica clínica con drogodependientes. Adicciones, 14(3), 345-370. Recuperado de http://www.adicciones.es/index.php/adicciones/article/view/490/486

Verdejo-García, A., Pérez-García, M., Bechara, A. (2006). Emotion, decision-making and substance dependence: a somatic-marker model of addiction. Current Neuropharmacology, 4(1), 17-31. Recuperado de https://www.ncbi.nlm.nih.gov/ pmc/articles/PMC2430678/

Verdejo, A., Toribio, I., Orozco, C., Puente, K. L., Pérez-García, M. (2005). Neuropsychological functioning in methadone maintenance patients versus abstinent heroin abusers. Drug and Alcohol Dependence, 78(3), 283-288. DOI: http://doi.org/10.1016/j.drugalcdep.2004.11.006 
Wang, P-W., Lin, H-C., Wu, H-C., Hsu, C-Y., Chung, K-S., Ko, C-H., Yen, C-F. (2015). Explicit and implicit heroin-related cognitions and heroin use among patients receiving methadone maintenance treatment. Comprehensive Psychiatry, 56, 155160. DOI: http://doi.org/10.1016/j.comppsych.2014.08.047

Yan, W-S., Li, Y-H., Xiao, L., Zhu, N., Bechara, A., Sui, N. (2014). Working memory and affective decision-making in addiction: A neurocognitive comparison between heroin addicts, pathological gamblers and healthy controls. Drug and Alcohol Dependence, 134(1), 194-200. DOI. http://doi.org/10.1016/j. drugalcdep.2013.09.027 